\title{
Adaptive Method for Tuning Dynamic Properties of Beams by Means of Particles Redistribution
}

\author{
M. ŻuRAWSKI* AND R. ZALEWSKI \\ Warsaw University of Technology, 02-524 Warsaw, Poland \\ Doi: $10.12693 /$ APhysPolA.138.240 \\ *e-mail: Mateusz.Zurawski@pw.edu.pl

\begin{abstract}
The paper concerns problems related to controlling the properties of vibrating slender elements. The main mechanism that influences global mechanical features of the system is redistribution of the grains located inside the considered element. The dynamic properties of the investigated system can be controlled by external factors resulting in partial changes in grains redistribution. An application of special pneumatic system allows to change either stiffness or mass distribution (density) of the beam. Original experimental results are presented in the paper. It is confirmed that there is a significant and direct connection between the granular mass distribution and the dynamics of the system. The spectral vibration analyses were carried out for various particles redistribution cases. Additionally an initial approach to the modeling process is introduced. The continuous model of cantilever beam has been simplified to a discrete multi DOF system. The verification of experimental and numerical data revealed the satisfactory convergence. It is confirmed that there is a possibility to find such parameters of the system which allow to avoid dangerous resonant frequencies.
\end{abstract}

topics: Damping of vibrations, mass redistribution, cantilever beam, Particles Impact Beam

\section{Introduction}

Vibrations are processes in which the selected physical quantities change over time. From the engineering point of view the most important are mechanical vibrations, defined as the movement of the elastic medium particles relative to the equilibrium position. Mechanical vibrations can spread in both liquid and solid media. However, in the aspect of human protection and safety only mechanical vibrations that are propagated in solid media are considered. Mechanical vibrations are often generated for the implementation of specific technological processes. Nevertheless, they often are a side effect that unintentionally accompanies the operation of machinery and equipment. In both cases, mechanical vibrations generated by various types of sources can cause damage to mechanical structures and interfere with their proper operation, as well as accelerate wear of components. In contact of a human with a vibrating element, vibrations are transmitted to his body, and can pose a threat to health and even life. Therefore, the damping of mechanical vibrations has been the subject of systematic observation and research for several decades.

Designing contemporary engineering structures increasingly requires dynamic loads to be taken into account. This requires the development of new strategies to attenuate structure vibrations that would not require an increase in the rigidity and/or mass of the structure. These strategies, called damping of vibrations methods, have developed intensively in the last thirty years. They are generally divided into passive, active and semi-active methods.

Recently, lot of interesting ideas for unwanted mechanical vibrations damping using granular media can be found in a world literature. An original method for determining vibration damping of the cantilever beam can be found in [1]. It involves an electromagnetic resonator that distinguishes the type of damping (internal, thermoelastic or aerodynamic).

Also innovative smart structures are used for the purpose of vibrations attenuation. Typical example of such structures can be Vacuum Packed Particles (VPP). They consist of loose grains that exhibit various mechanical properties according to the range of internal pressure [2]. VPP can be an efficient solution in semi-active damping of beam like elements vibration. In such cases the underpressure was a convenient factor to change the dissipative properties of the system.

An interesting study is presented in [3] where a damper is treated as a double pendulum striking element. This seems to be especially effective for damping of slender elements vibrations. For example, in [4-9] the particles are used to damping mechanical systems (Particles Impact Dampers, PID) and they are treated as a gas flow. In a consequence, the damping coefficients are described by formulas dependent on the restitution coefficient, excitation frequency, mass ratio and particles density. 
In [10], the PID damper parameters are identified by Artificial Neural Network. The effectiveness of presented solution is compared with two other methods, i.e., Feed Forward Back Propagation Network and Radial Basis Function.

It seems that granular materials can be applied to attenuate vibrations of brittle electric plates of the spacecrafts during the flight [11]. PID can be an example of the energy harvesting application [12]. Particles are replaced by a coil magnet which can move in the container and introduce new forces in the global damping system. A lot of vibrations problems can be observed in the wind turbines. In [13-16] authors proposed the structure with grains to attenuate undesirable dynamical effects. The other papers reveal modified PID structures [17-22]. They are based on Chain and Vibro-Impact Nonlinear Energy Dampers or sound absorbers.

Very often smart materials are incorporated in semi-active damping of vibration strategies. In fact, papers $[23,24]$ show the possibility of using MR elastomer in the design of honeycomb beams. The importance of the structural damping problem and its practical usefulness is presented. The experiments show the influence of magnetic field on the dynamics of the systems. Described methods allow to shift easily the natural frequencies of vibrations.

This paper is devoted to introducing a novel method for slender objects vibrations attenuation. The dynamics of the vibrating beam element is in real time controlled by a special pneumatic system enabling the granular material being placed inside the system to be redistributed. The proposed method of the damping of vibrations can be placed among novel strategies of semi-active vibration attenuations. To reveal extraordinary features of the discussed concept a typical cantilever beam subjected to kinematical excitation rule was used.

\section{Test stand}

The test stand consists of a kinematic exciter and a specially prepared beam with granular sections. The engine drives subsequent mechanical elements, and the rotary movement is converted into a reciprocating movement resulting in demanded kinematic excitation of the fixed end of the investigated beam (Fig. 1). In the experiments the constant value of the excitation amplitude $(5 \mathrm{~mm})$ was assumed. In the laboratory tests various frequencies (in the range $0-50 \mathrm{~Hz}$ ) were taken into account. The left end of the investigated beam is fixed in a special handle and attached to the exciter.

The laboratory stand also involves the air compressor with a distribution valve system that allows user to change the position of the previously mentioned granular section. The measuring system consists of a displacement laser sensor located at the free right end of the beam, a personal computer with a measuring card and a data analyzer.

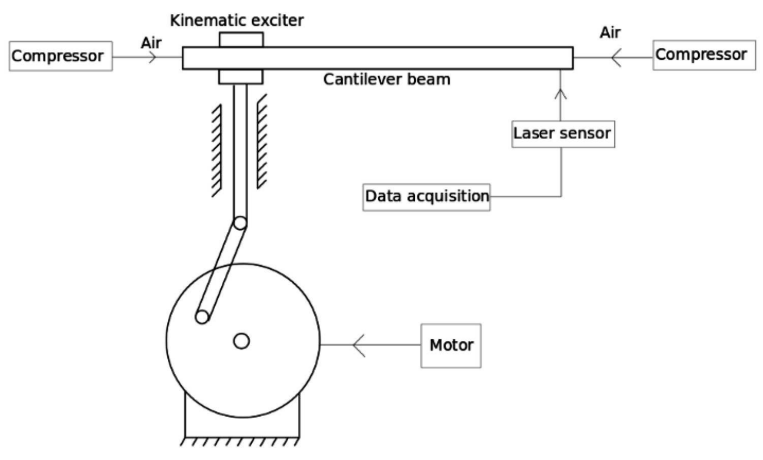

Fig. 1. Scheme of the test stand.
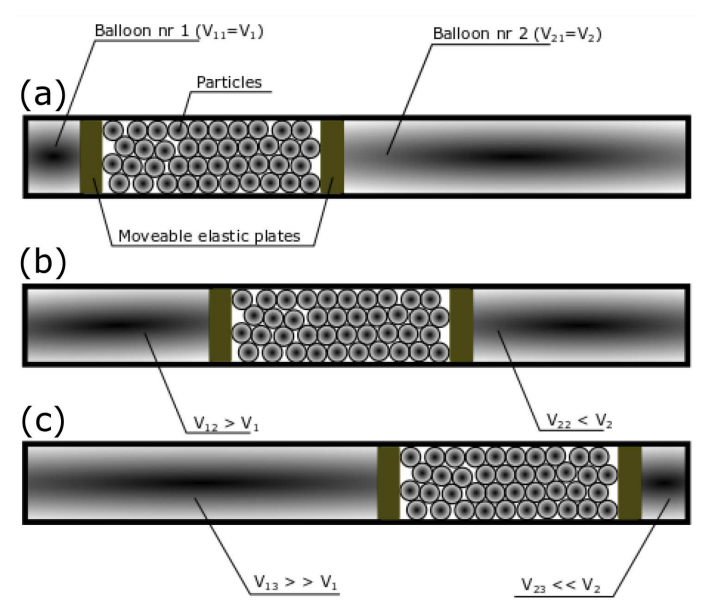

Fig. 2. Investigated object.

The tested object is a beam (Fig. 2) having a rectangular cross-section $(a=50 \mathrm{~mm}, \quad b=30 \mathrm{~mm})$ with free space inside along its entire length $(l=500 \mathrm{~mm})$. This space has been divided into three sections through flexible sliding discs. The outer chambers are equipped with balloons, and changing their volume (inflating or deflating) enables changing the position of flexible disks. In the central section the plastic ball-shaped granular material is located. The weight of the granulate is $40 \%$ of the total weight of the system. Inflating and deflating the balloons results in displacements of internal discs together with granular material placed between them. Such operation results in two important phenomena: internal mass redistribution and local increase of the beam stiffness. Both phenomenon have a strong impact on the global dynamics of the investigated cantilever beam.

At this preliminary stage of research, it is assumed that the particles are enclosed in a central section, locking their movement in a tangential and normal direction. Three cases of granular section localization were investigated in the experiments: close to the fixed left end (Fig. 2a), in the middle (Fig. 2b) and close to the right free end (Fig. 2c). As it was previously mentioned the beam is excited in a harmonic way and the displacement of the free end of the beam is measured. The exciting 


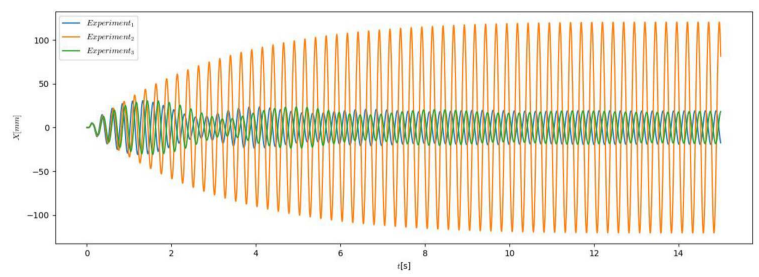

Fig. 3. Comparison of deflections in three different granular section positions.

frequency was constant $(f=3.98 \mathrm{~Hz})$ and corresponded to the resonant frequency for the case when the granular section is located in the middle of the beam. Beam responses for all three cases are depicted in Fig. 3.

Analyzing the results depicted in Fig. 3, it can be observed that shifting the granular mass from the middle section to the beginning (Experiment $\left.{ }_{1}\right)$ or end of the beam (Experiment ${ }_{3}$ ) effectively reduces the vibration amplitude of the investigated object, which is initially in resonance $\left(\right.$ Experiment $\left._{2}\right)$. In this case, the aspect of changing the natural frequency of the system was achieved simply by shifting the resonance range by changing the mass in a specific section. The phenomenon is a little bit more complicated because of the fact that in the considered section also the local stiffness, resulted from the compaction of the grains, is increased.

The novelty on which the authors want to focus in this paper is the ability to quickly change these parameters and adapt them to the nature of the work of the vibrated system. In addition, with some modifications, the proposed solution can be used to attenuate vibrations of rotating elements.

\section{Modelling}

The applied at this stage of investigations mathematical model of the tested system is a simple $6 \mathrm{DOF}$ model. It was assumed that the global mass of the system (including granular materials) was uniformly distributed in six nodes. Additional assumptions are: the same damping properties for each degree of freedom and the small stiffness variations in selected sections $( \pm 5 \%)$ resulting from the various compactions of grains.

Thus the formula describing energy of the system is a function of mass and stiffness coefficients and can be defined as:

$$
\begin{aligned}
& E_{p}=\sum_{i=1}^{n} E_{p_{n}}\left(k_{n}, x_{n}\right), \\
& E_{k}=\sum_{i=1}^{n} E_{k_{n}}\left(m_{n}, \dot{x}_{n}\right),
\end{aligned}
$$

where $n$ - number of degrees of freedom, $x$ is free right end of the beam displacement, and $\dot{x}-$ velocity of the beam tip.
As a result, the equation of motion of the considered model including kinematic excitation can be presented as:

$$
\begin{aligned}
& M \ddot{x}+C_{1} \dot{x}+K_{1} x=C_{2} \dot{u}+K_{2} u, \\
& u=U \sin \left(2 \pi f_{\text {res }} t\right),
\end{aligned}
$$

where $M, C_{1}, C_{2}, K_{1}, K_{2}$ are the matrixes of mass, damping and stiffness respectively, $U$ is excitation amplitude, $f_{\text {res }}$ - excitation frequency equal to the natural frequency of the system for the case where the grains are located in the beam center.

Due to the preliminary stage of research the simplest numerical identification procedure was used to calibrate the mathematical model. The unknown values of system parameters (stiffness and damping matrixes components) were determined by the Monte Carlo method where the optimization function was defined as the absolute sum of the differences between the values of experimental and numerical displacements (5). The algorithm in 1000 iterations is looking for the optimal $K$ and $C$ matrixes for which the optimization function reaches the minimum. The additional limitation was the initial assumption that the mass redistribution to specific nodes changes the stiffness of this section by a maximum of $5 \%$. One can write

$$
E=\sum_{i=1}^{N}\left|x^{\exp }\left(t_{i}\right)-x^{\operatorname{sim}}\left(t_{i}, k_{n}, c_{n}\right)\right| \rightarrow \min ,
$$

where $k_{n}$ and $c_{n}$ is a set of equivalent parameters of stiffness and damping for a system with $n$-degrees of freedom.

Even the simple Monte Carlo strategy has effectively identified the $K$ and $C$ parameters. Basing on the data depicted in Figs. 4-6, it can be stated that quite a good convergence between real experimental data and numerical simulation results was observable. Conducted analysis revealed that for stabilized vibrations the proposed modeling method seems to be sufficient at the initial stage of theoretical studies of the proposed mass redistribution damping of vibrations strategy.

The experimental tests revealed the applicability of the proposed method in damping of the investigated beam resonance vibrations. However, it should be emphasized that any form of mass redistribution causes the passage (usually rapid) through the resonance range, which in effect will always

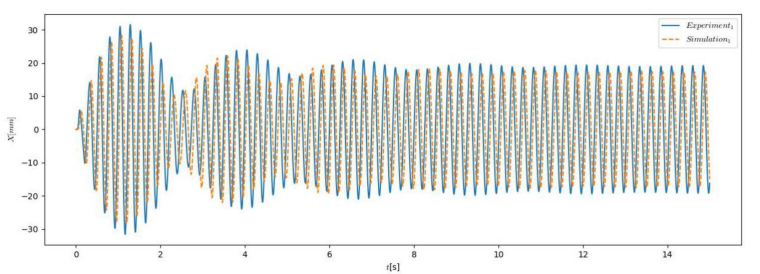

Fig. 4. Verification of experimental and numerical results for the granular section located close to the fixed end of the beam. 


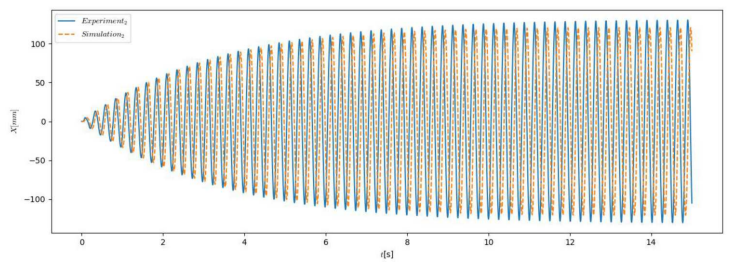

Fig. 5. Verification of experimental and numerical results for the granular section located in the middle of the beam.

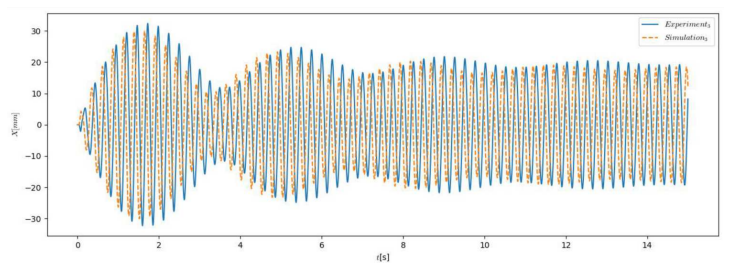

Fig. 6. Verification of experimental and numerical results for the granular section located close to the free end of the beam.

cause a temporary increase in the amplitude of vibrations. Therefore, one should consider the possibility of reducing vibrations in the resonance range itself. The method discussed in the paper is based on moving the granular section by uniformly inflating one balloon and deflating the other.

Assuming that the sliding discs inside the beam are moving with various velocities or in opposite directions the granular materials inside the system will not be compacted anymore (Fig. 7). They will start to collide with each other and with the beam interior. Such a situation will lead to additional energy dissipation related to nonlinear character of the mentioned "intergranular" contacts with friction. Such a construction can be described as a Particles Impact Beam (PIB), i.e., a beam equipped with an additional impact damper. In the widely described literature one can find a large applicability of this type of Particles Impact Dampers PIDs. It turns out that they generally operate within the frequencies close to the resonance range. In Fig. 8, a comparison of experimental tests for the case when the granular material is compressed and located in the central part of the beam (Fig. 2b) and the case when the particles are "loose" inside the system-PIB (Fig. 7) is depicted.

Analyzing the data presented in Fig. 8, one can observe a decrease in vibration amplitude by nearly $50 \%$ in the resonance range when the granulate acts as a PID. It should be emphasized that the natural frequencies in both cases of particles redistribution were the same, which confirms that the additional damping in the beam can be activated.

In order to move the granular structure to a specific section, it is necessary to take into account the situation when the accelerating system will be forced with a frequency close to the natural frequency of the beam. Then the granulate

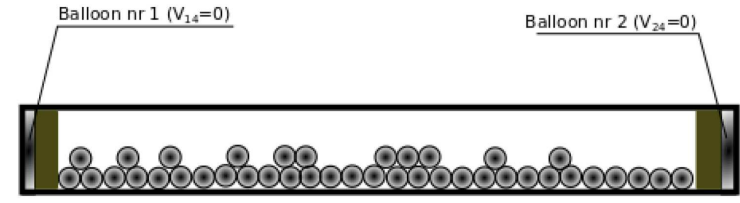

Fig. 7. Particles Impact Beam — PIB.

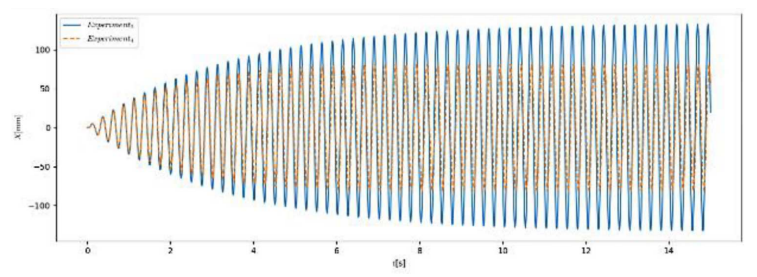

Fig. 8. Comparison of experiments - granular section vs. PIB.

should be temporarily spread over the entire beam. In a situation where the excitation frequency exceeds the resonance range, the controlled balloons inflating/deflating process can be activated to redistribute the granular section to a specific region of the beam, for which the natural frequency will be significantly different from the external excitation frequency at which the tested object will operate.

\section{Conclusions}

In the paper the novel method for the adaptivepassive (semi-active) damping of vibrations is presented. It is related to the controlled mass of the system redistribution using movable granular material. Thanks to two balloons placed inside the slender element, the loose grains can be freely shifted along the beam axis. The movement of the grains results in controlled inflating and deflating strategy of the balloons. The conducted experiments confirmed the effectiveness of the presented method of shaping the dynamical properties of the investigated system. The controlled mass redistribution method can be rapidly transformed into classical PID device. The proposed innovative method for damping of vibrations opens up new possibilities for interesting engineering applications. They seem to be specially interesting for slender structures like wing turbine blades or similar long light beam like structures.

\section{References}

[1] Faruq Muhammad Foong, Chung Ket Thein, D. Yurchenko, Mech. Sys. and Sig. Proc. 119, 120 (2018).

[2] J.M. Bajkowski, R. Zalewski, Mech. Res. Comm. 60, 1 (2014).

[3] P. Egger, L. Caracoglia, J. Sound Vib. 353, 38 (2015). 
[4] F. Djemal, R. Chaari, W. Gafsi, F. Chaari, M. Haddar, Appl. Acou. 147, 72 (2019).

[5] K.S. Marhadi, V.K. Kinra, J. Sound Vib. 283, 433 (2005).

[6] C. Snoun, M. Trigui, Int. J. Interact. Des. Manuf. 12, 1283 (2018).

[7] E. Sonmez, S. Nagarajaiah, C. Sun, B. Basu, J. Sound Vib. 362, 1 (2016).

[8] M. Sanchez, C. Manuel Carlevaro, J. Sound Vib. 332, 2070 (2013).

[9] M. Sanchez, L.A. Pugnaloni, J. Sound Vib. 330, 5812 (2011).

[10] P. Veeramuthuvel, K. Shankar, K. Sairajan, R. Machavaram, Proc. Mater. Sci. 5, 335 (2014).

[11] P. Veeramuthuvel, K. Shankar, K. Sairajan, Acta Astronaut. 127, 260 (2016).

[12] A. Afsharfard, Mech. Syst. Signal Process. 98, 371 (2018).

[13] B. Fitzgerald, S. Sarkar, A. Staino, J. Sound Vib. 419, 103 (2018).

[14] M. Hussan, M.S. Rahman, F. Sharmin, D. Kim, J. Do, Ocean Eng. 160, 449 (2018).
[15] C. Junling, G. Christos, J. Sound Vib. 332, 5271 (2013).

[16] Z. Zhang, A. Staino, B. Basu, S.R. Nielsen, Eng. Struct. 126, 417 (2016).

[17] O. Gendelman, J. Sound Vib. 331, 4599 (2012).

[18] O. Gendelman, A. Alloni, Procedia IUTAM 19, 53 (2016).

[19] M. Gharib, S. Ghani, J. Sound Vib. 332 , 6254 (2013).

[20] M. Gharib, M. Karkoub, M. Ghamary, Mech. Syst. Signal Process. 3, 34 (2016).

[21] L. Hu, Y. Shi, Q. Yang, G. Song, J. Sound Vib. 384, 45 (2016).

[22] T. Li, E. Gourc, S. Seguy, A. Berlioz, Int. J. Non-Lin. Mech. 90, 100 (2017).

[23] A. Boczkowska, S.F. Awietjan, S. Pietrzko, K.J. Kurzydlowski, Compos. B: Eng. 43, 636 (2012).

[24] Felipe Souza Guilherme Gomesa, Compos. Struc. 176, 231 (2018). 